\title{
Reversible acute kidney injury requiring haemodialysis five days after starting dronedarone in a stable 7I-year-old man at risk of cardiovascular polypharmacy
}

\author{
${ }^{1} \mathrm{C}$ Young, ${ }^{2} \mathrm{M}$ Maruthappu, ${ }^{3} \mathrm{RP}$ Wayne, ${ }^{4} \mathrm{~L}$ Leaver \\ ${ }^{1}$ FY1, St James's University Hospital, Leeds; ${ }^{2} 6$ th Year Medical Student, Green Templeton College, University of Oxford; ${ }^{3}$ Emeritus Professor of \\ Chemistry, University of Oxford; ${ }^{4}$ Senior Joan $\mathcal{E}$ Richard Doll Clinical Tutorial Fellow, Green Templeton College, University of Oxford, UK
}

\begin{abstract}
We present a case of severe acute kidney injury (AKI) occurring shortly after the initiation of dronedarone therapy, which we suspect was the result of an adverse drug reaction. The mechanism of AKI cannot be definitively determined. The most probable mechanism however involves dehydration secondary to diarrhoea, and medications causing hypotension, both precipitating AKI, further exacerbated by reduced excretion of medications reducing tissue perfusion. This case adds to the growing number of reports submitted to pharmacovigilance authorities regarding the association between dronedarone and AKI. It serves as a reminder of the risks of cardiovascular polypharmacy likely to be prevalent in patients considered for dronedarone (which causes diarrhoea as a common side-effect).
\end{abstract}

\author{
Correspondence to L Leaver, \\ Senior Joan \& Richard Doll \\ Clinical Tutorial Fellow, \\ Green Templeton College, \\ Oxford OX2 6HG, UK
}

tel. $+44(0) 18653$ II 234

e-mail

laurence.leaver@gtc.ox.ac.uk

KEYWORDS Dronedarone, acute kidney injury, adverse reaction, case report, pharmacovigilance

DECLARATION OF INTERESTS No conflicts of interest declared.

\section{INTRODUCTION}

A 7I-year-old man suffered acute kidney injury (AKI) five days after commencing dronedarone. This drug is indicated for non-sustained atrial fibrillation (AF). It reduces creatinine clearance' by specific competitive partial inhibition of tubular organic cation transporters, but is not thought to otherwise impair glomerular filtration rate (GFR) or kidney function. ${ }^{2}$ This case, however, adds to a growing number of reports of renal impairment associated with dronedarone.

Medicines and Healthcare Products Regulatory Agency (UK) (MHRA) data regarding dronedarone ${ }^{3}$ describe two reports of renal failure, two of acute renal failure, six of renal impairment, one of anuria, one of oliguria, nine of raised creatinine, three of decreased GFR, two of reduced creatinine clearance, and one of raised urea. 'Eudravigilance' worldwide data contained 44 reports of $\mathrm{AKI}$ and 49 reports of renal failure by June $201 \mathrm{I}$ (Ana Hildago-Simon, European Medicines Agency June, 20 I I). The US Food and Drug Administration (FDA) received at least 15 reports of kidney impairment by November $2010,{ }^{4}$ and recommends monitoring of renal function. ${ }^{5} \mathrm{~A}$ recent paper $^{6}$ reviewed 124,069 adverse drug reaction (ADR) reports from Italy between October 2010 and December 201 I; dronedarone was listed as a suspected drug in 55 cases, and among these were reported four cases of acute renal failure (ARF), two of renal failure
(RF) and three cases of increase of blood creatinine (although details of each case are limited).The Magdeburg Dronedarone Registry (MADRE) study took a more detailed prospective approach to studying the adverse reactions associated with dronedarone. They found three out of 191 patients experienced $>40 \%$ increase in creatinine concentration. ${ }^{?}$

A Medline search (using the terms 'dronedarone' and 'acute kidney injury' or 'renal impairment' in February 2013) yielded only one case report of renal impairment associated with dronedarone; ${ }^{8}$ this impairment was considered secondary to aggravation of pre-existing heart failure. Searching references from reviews, other articles and non-academic internet searches, we only found reports with limited additional data, from the Uppsala Monitoring Centre. ${ }^{9}$ Our case report is the first to detail an association between $\mathrm{AKI}$ and dronedarone in a previously stable patient.

\section{CASE REPORT}

The patient had symptomatic paroxysmal AF for 24 years, treated with sotalol and digoxin. He requested dronedarone to reduce symptomatic episodes of AF. His cardiologist devised a protocol to initiate dronedarone which was compatible with NICE guidelines ${ }^{10}$ and the Summary of Product Characteristics" at the time, even though it may not be a typical practice in the UK. 
The patient's past medical history included coronary artery bypass grafting 12 years before, hypertension for 34 years, hypercholesterolaemia for 20 years, type 2 diabetes mellitus for five years, gout for 20 years and benign prostatic hypertrophy for nine years. All were well-controlled. There were no symptoms, signs or history of heart failure. All previous electrocardiograms (ECGs) and Holter ECGs were normal apart from the paroxysmal AF. Echocardiography, both before and after recovery, was normal. Serum digoxin concentrations were not abnormally high prior to the episode.

Medications prior to the replacement of sotalol with dronedarone were: atorvastatin (40 mg once daily [od]), allopurinol (300 mg od), finasteride (5 mg od), metformin (500 mg four times a day [qds]), Gaviscon Advance (I0 $\mathrm{ml}$ as needed [prn]), sotalol (80 mg qds), Omacor (I,000 $\mathrm{mg}$ od), candesartan (32 mg od), doxazosin (4 mg od), spironolactone ( $5 \mathrm{mg}$ od), Sando-K ( $12 \mathrm{mmol} \mathrm{K}^{+}$twice a day [bd]), warfarin ( $3.75 \mathrm{mg}$ od according to international normalised ratio [INR]), and digoxin (250 $\mu g$ od). The patient had no drug allergies, took no proprietary or complementary medicines (including non-steroidal anti-inflammatory drugs [NSAIDs]) and he didn't drink grapefruit juice.

The patient was Caucasian and a retired professor. His height was $1.7 \mathrm{~m}$, he weighed $92 \mathrm{~kg}$ and he was an ex-smoker, for 13 years. His alcohol intake was approximately 42 units/week. There was no relevant family history. The day-to-day progress of the case is detailed in Figures I and 2. The case was reported through the UK Yellow Card scheme for reporting adverse drug reactions and to the manufacturer of dronedarone.

\section{DISCUSSION}

Acute kidney injury became apparent five days after dronedarone therapy was commenced. No other new drugs were given except bisoprolol (from day two) to replace sotalol (it had been tolerated by the patient four years earlier). The patient was previously well, without evidence of other acute illness. All symptoms improved after dronedarone was stopped and haemodialysis performed. It is unknown whether withdrawal of dronedarone without haemodialysis would have reversed the AKI. The manufacturer's prescribing information states that it is not known whether dronedarone is removed by dialysis."

The mechanism by which dronedarone might have caused AKI is not established. Urinary osmolality, fractional sodium excretion and microscopy results were not available. Possible renal mechanisms include direct nephrotoxicity, indirect nephrotoxicity or interstitial nephritis. However, the most probable mechanism is that of pre-renal AKI.
It is likely that a combination of dehydration (secondary to diarrhoea), dronedarone and the other medications reducing tissue perfusion led to hypotension and bradycardia. Low blood pressure and pulse rate would have caused renal hypoperfusion and subsequently reduced excretion of the medications. There is thus a positive feedback between $\mathrm{AKI}$, hypotension and bradycardia. This is not an uncommon risk of cardiovascular polypharmacy. ${ }^{12}$

There are several aspects unique to this case. Diarrhoea could have been a side-effect of dronedarone (as is commonly the case), but the INR was raised on day six, prior to dronedarone initiation and prior to diarrhoea. This elevation of the INR could have been due to another process, perhaps also causing changes in renal function. Warfarin dosing is usually only minimally affected by renal function. The INR could have been altered via cytochrome P450 changes caused by altering the patient's regular medications (replacement of sotalol with bisoprolol and halving of the digoxin dose) prior to starting dronedarone.

The hypomagnesaemia also remains unexplained. It persisted after haemodialysis, despite resolution of the diarrhoea, a reduction in alcohol intake and oral and IV supplementation. Phosphate, calcium and parathyroid hormone (PTH) were normal. Fractional excretion of magnesium was normal but arguably inappropriately high for the plasma level, suggesting renal wasting (possibly indicative of anomalous tubular function unrelated to this episode).

These observations are difficult to explain, and exemplify why analysis of data from multiple reports is necessary in order to have greater confidence in any trends and potential mechanisms that may be proposed. It is unfortunate that pharmacovigilance authorities cannot release details of reported cases to allow analysis by independent experts. One could argue that the benefit to society might justify a requirement to provide detailed information (subject to appropriate safeguards or obtaining consent for disclosure) similar to the reporting of notifiable diseases or compulsory disease registers in some countries. Prospective studies (such as the MADRE study) are welcome but are difficult to conduct with very large numbers of patients.

\section{CONCLUSION}

A 7I-year-old man experienced reversible $\mathrm{AKI}$, temporally associated with starting dronedarone. The mechanism was probably pre-renal, but cannot be proven definitively. It serves as a reminder of the risks of cardiovascular polypharmacy, common to the subset of patients using dronedarone, combined with diarrhoea (a recognised side-effect of the drug). It is reassuring that 
Day I: The day sotalol was stopped; other medication changes were made as recommended by the Summary of Product Characteristics. ${ }^{8}$ The eGFR was calculated using the modification of diet in renal disease (MDRD) method.

Day 6: Uncharacteristic INR of 3.6, previous baseline 2.0. Cause unknown.

Day 7: Dronedarone commenced.

Day 8: Regular bradycardia $30 \mathrm{bpm}$. The patient had no evidence of sinus node disease - previous ECG and Holter ECGs were normal. Home blood pressure (BP) of 65/40 $\mathrm{mmHg}$ (compared to average home BP I I 2/55 mmHg and pulse rate of $43 \mathrm{bpm}$ in the week preceding discontinuation of sotalol). Diarrhoea, oliguria, dizziness, nausea, abdominal pain.

Day 9: ECG sinus bradycardia, 30 bpm.
Day 10: Patient, concerned by oliguria (approximately $10 \mathrm{~mL} / 12$ hours), drank $2 \mathrm{~L}$ oral rehydration solution. No symptoms of urinary retention. Light coloured urine. Patient noted weight gain.

Day I I: Digoxin and dronedarone discontinued (after eight doses); patient's decision.

Day 12: Patient presented to GP. Admitted to hospital with $\mathrm{AKI}$, oliguria, oedema, xanthopsia (probably secondary to digoxin toxicity). Normal ECG and liver function tests (LFTs). Blood tests (Figure 2). All medications discontinued. Management included salbutamol (nebulised), calcium gluconate (IV), bicarbonate (IV).

Day 14: AKI still unresolved. Haemodialysis (once).

Renal function remained acceptable post-dialysis. Magnesium was found to be low pre-dialysis and has remained low subsequently despite oral supplements but no cause was found.

FIGURE I Day-to-day events in the progress of the case.

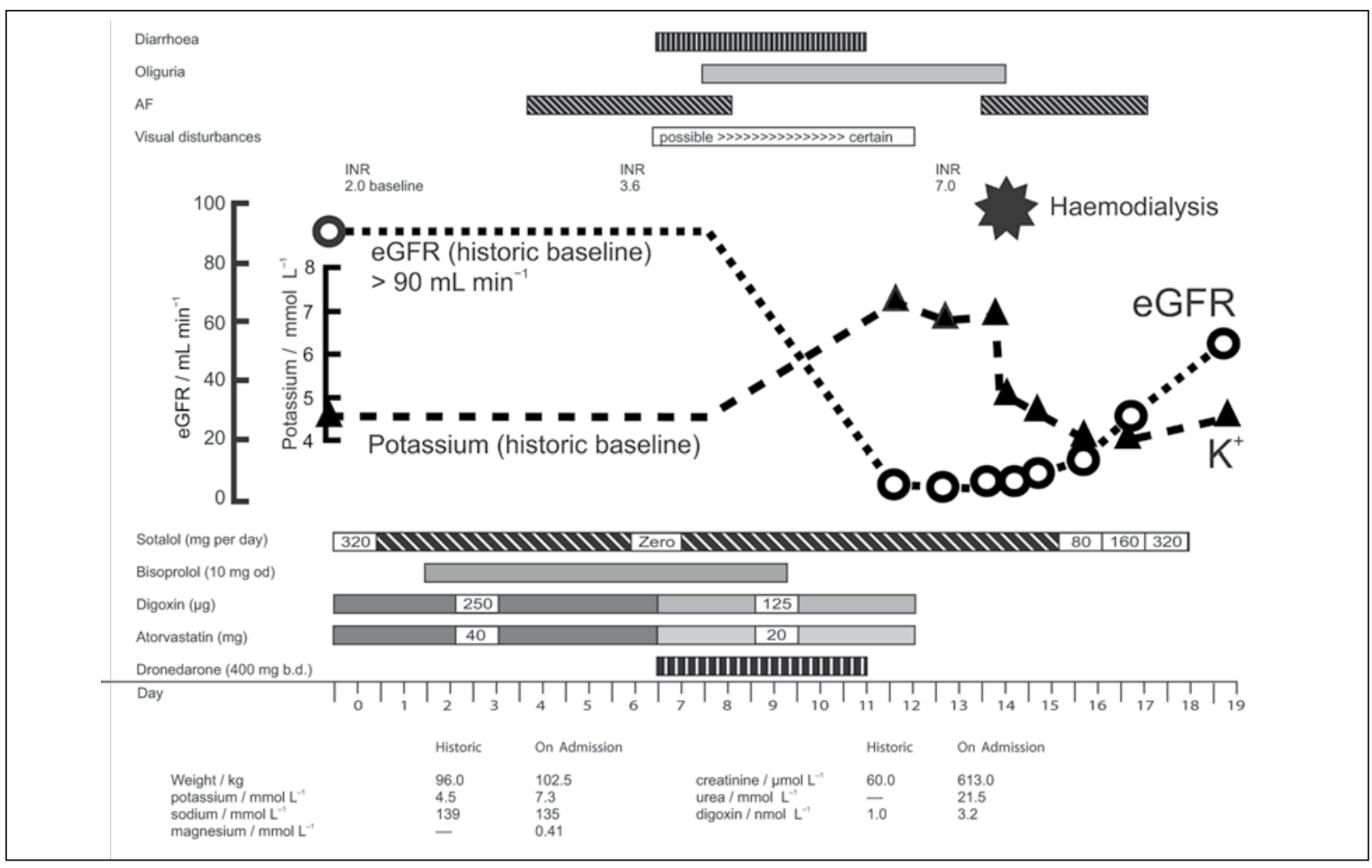

FIGURE 2 The relative timing of various events in this case report.

The abscissa represents the number of days after a nominal 'day zero'; the line above it shows the period over which the patient took dronedarone, and the lines at the top indicate the symptoms reported by him. Detailed information about the drug dosages and changes in them appear under the graphical portion. The replacement of sotalol by bisoprolol over days $1-2$, and the halving of the doses of atorvastatin and digoxin on day 7, when dronedarone therapy started, are displayed. The points and lines on the graph show the evolution of estimated glomerular filtration rate (eGFR) (calculated from plasma creatinine concentrations using the modification of diet in renal disease [MDRD] method) and the plasma concentrations of potassium. Very low eGFR and high $\left[\mathrm{K}^{+}\right]$appear within a few days of inception of therapy with dronedarone, and more normal values are restored immediately (a few hours) after haemodialysis on the evening of day 14 (represented here as abscissa value day 14.5). The patient's usual doses of medication were reintroduced slowly and cautiously from day 15. 
AKI was not found in the main trials of dronedarone." This case report must be seen in the context of widespread use of dronedarone. It adds to the number of reports submitted to pharmacovigilance authorities.

\section{Acknowledgement}

The authors wish to thank Dr Yaver Bashir for the valuable cardiological advice he gave concerning the management of the case and in the preparation of this report. They also wish to thank Dr Pete Biggs for preparing the final version of Figure 2.

\section{REFERENCES}

I Tschuppert Y, Buclin T, Rothuizen L et al. Effect of dronedarone on renal function in healthy subjects. Br J Clin Pharmacol 2007; 64:785-9l.

2 Duncker D, Oswald H, Gardiwal A et al. Stable cystatin C serum levels confirm normal renal function in patients with dronedaroneassociated increase in serum creatinine. J Cardiovasc Pharmacol Ther 18: 109-12. http://dx.doi.org/I0. I I77//0742484/2453873

3 Medicines and Healthcare Products Registry Agency. Drug analysis print [Internet]. London: MHRA; 2013 [cited 2013 Feb I3].Available from: http://www.mhra.gov.uk/home/groups/public/documents/ sentineldocuments/dap_I363951919050.pdf

4 The Institute for Safe Medication Practices. QuarterWatch: 2010 Quarter I Monitoring Med-watch Reports. Signals for acetaminophen, dronedarone and botulinum toxin products. [Internet]. Pennsylvania: ISMP; 2010 [cited 2012 July 25]. Available from: http://www.ismp. org/QuarterWatch/20I0QI.pdf

5 US Food and Drug Aministration. Risk evaluation and mitigation strategy for multaq, modified June 13, 2012. [Internet]. Maryland: FDA; 2009 [cited 2012 July 25]. Available from: http://www.fda.gov/downloads/Drugs/ DrugSafety/PostmarketDrugSafetylnformationforPatientsandProviders/ UCMI87494.pdf

6 Biagi C,Venegoni M, Melis M et al. Dronedarone-associated acute renal failure: evidence coming from the Italian spontaneous ADR reporting database. $\mathrm{Br} J$ Clin Pharmacol 2012; 17: Epub ahead of print. http://dx.doi.org// 0. I I I I/bcp. 12005

7 Said SM, Esperer HD, Kluba K et al. Efficacy and safety profile of dronedarone in clinical practice. Results of the Magdeburg Dronedarone Registry (MADRE study). Int J Cardiol 2012; Forthcoming. http://dx.doi.org/I0.1016/j.ijcard.20I2.06.056

8 Coons J, Plauger K, Seybert AL et al.Worsening heart failure in the setting of dronedarone initiation. Ann Pharmacother 2010;44:1496500. http://dx.doi.org/ I0.I345/aph.IP075

9 Savage RL. Dronedarone - acute renal failure and decreased creatinine clearance. In: WHO Collaborating Centre for International Drug Monitoring. Signal analyses of reports in the WHO global ICSR database - vigibase. [Internet]. Sweden: Uppsala Monitoring Centre; 2010 [cited 2012 July 25]. Available from: http://www.otago.ac.nz/christchurch/otago032053.pdt

10 National Institute for Health and Clinical Excellence. Dronedarone for the treatment of non-permanent atrial fibrillation. NICE Technology Appraisal Guidance 197, August 2010. [Internet]. London: NICE 2010 [cited 2012 July 25]. Available from: http://www.nice.org.uk/ nicemedia/live/ I 3 I 15/50469/50469.pdf

II European Medicines Agency. Summary of product characteristics (multaq). [Internet]. London: EMA [cited 20II Aug 2I]. Available from: http://www.ema.europa.eu/docs/en_GB/document_library/ EPAR_-_Product_Information/human/00 I043/WC500044534.pdf

12 Berry C, McMurray JJ. Serious adverse events experienced by patients with chronic heart failure taking spironolactone. Heart 200I; 85:E8. http://dx.doi.org/I0.II36/heart.85.4.e8 